\title{
Nesting symmetries and diffusion in disordered $d$-wave superconductors
}

\author{
A. G. Yashenkin ${ }^{1,2, \dagger}$, W. A. Atkinson ${ }^{1,3}$, I. V. Gornyi ${ }^{4, \ddagger}$, P. J. Hirschfeld ${ }^{1}$, and D. V. Khveshchenko ${ }^{2}$ \\ ${ }^{1}$ Department of Physics, University of Florida, PO Box 118440, Gainesville FL 32611 \\ ${ }^{2}$ Department of Physics and Astronomy, University of North Carolina, Chapel Hill, NC 27599 \\ ${ }^{3}$ Department of Physics, Southern Illinois University, Carbondale, IL 62901-4401 \\ 4 Institut für Nanotechnologie, Forschungszentrum Karlsruhe, 76021 Karlsruhe, Germany
}

(October 29, 2018)

\begin{abstract}
The low-energy density of states (DOS) of disordered 2D $d$-wave superconductors is extremely sensitive to details of both the disorder model and the electronic band structure. Using diagrammatic methods and numerical solutions of the Bogoliubov-de Gennes equations, we show that the physical origin of this sensitivity is the existence of a novel diffusive mode with momentum close to $(\pi, \pi)$ which is gapless only in systems with a global nesting symmetry. We find that in generic situations, the DOS vanishes at the Fermi level. However, proximity to the highly symmetric case may nevertheless lead to observable non-monotonic behavior of the DOS in the cuprates.
\end{abstract}

Introduction. An understanding of the quasiparticle (QP) excitations in the $d$-wave superconducting state of the high- $T_{c}$ superconductors is essential for the elucidation of transport properties, for determining how the ground state deviates from the BCS model, and for describing the instability of the lightly doped antiferromagnetic state to superconductivity. It has been known for some time that the influence of disorder on the QP states is quite different from ordinary superconductors, in part due to the gap symmetry and in part due to low dimensionality. Nersesyan et al. have shown that these two features conspire to introduce logarithmic divergences in all orders of the perturbation theory 11. Since then, several groups have attempted nonperturbative treatments of the "2D dirty $d$-wave problem", arriving at a surprisingly diverse set of results. The proposed scenarios predict vanishing [1 3], constant [4.5], and divergent [6.7] density of states (DOS) $\nu(\epsilon)$ as $\epsilon \rightarrow 0$ (energies are measured from the Fermi level) for apparently similar models. Recently, two of the authors argued [8] on the basis of numerical studies that the $d$-wave superconductor is fundamentally sensitive to "details" of disorder, as well as to certain symmetries of the normal state Hamiltonian. While this approach was successful in unifying the various analytical treatments, it failed to provide a physical explanation of the origin of this lack of robustness.

In this work, we combine perturbative analytical and numerical calculations with the intent of clarifying the physics of the various asymptotic results for the DOS of noninteracting QPs. We show that the generic result is $\nu(\epsilon \rightarrow 0) \rightarrow 0$, but that both the constant and divergent DOS can be obtained in the presence of global "nesting" symmetries (GNSs) which produce additional diffusive modes with momenta close to $\mathbf{Q}=(\pi, \pi)$ [9]. These symmetries drastically change the low-energy DOS and are explicitly obeyed by models considered in Refs. [4 and [6] where the constant and the divergent $\nu(\epsilon \rightarrow 0)$ have been found. The GNS relations necessary for the $\pi$-modes do not occur explicitly in real cuprates, but we demonstrate that even proximity to global nesting has observable consequences on thermodynamic properties.

Models of pure d-wave superconductor. The weaklocalization (WL) calculations will be compared directly with exact solutions of the Bogoliubov-de Gennes equations on finite tight-binding lattices, details of which have been published elsewhere 8, 10, We investigate three different model band structures, each having specific symmetry properties. The nearest neighbor (N1) model with normal-state dispersion $\xi_{\mathbf{k}}=-2 t\left(c_{x}+c_{y}\right)-\mu$, where $t$ is the hopping matrix element and $\mu$ is the chemical potential, is the most extensively studied. Here $c_{x, y}=\cos \left(k_{x, y} a\right)$ and $a$ is the lattice constant. We also consider a model including second-neighbor (N2) hopping, $\xi_{\mathbf{k}}=-2 t\left(c_{x}+c_{y}\right)-4 t^{\prime} c_{x} c_{y}-\mu$ and a peculiar thirdneighbor (N3) hopping model $\xi_{\mathbf{k}}=-4 t^{\prime \prime}\left(c_{x}^{2}+c_{y}^{2}-1\right)-\mu$. In all cases, the $d$-wave Hamiltonian assumes pairing between nearest neighbour sites, $\Delta_{\mathbf{k}}=\Delta_{0}\left(c_{x}-c_{y}\right)$. The lines of zeros for $\Delta_{\mathbf{k}}$ intersect the Fermi surface $\xi_{\mathbf{k}}=0$ at four symmetric nodal points for both the N1 and $\mathrm{N} 2$ models, and at eight points for the N3 model. In the vicinity of these points we may make an expansion of the QP spectrum $\varepsilon_{\mathbf{k}} \simeq\left[\left(\mathbf{v}_{F} \tilde{\mathbf{k}}\right)^{2}+\left(\mathbf{v}_{g} \tilde{\mathbf{k}}\right)^{2}\right]^{1 / 2}$, where $\mathbf{v}_{F}=\left(\partial \xi_{\mathbf{k}} / \partial \mathbf{k}\right)_{\mathbf{k}_{n}}, \mathbf{v}_{g}=\left(\partial \Delta_{\mathbf{k}} / \partial \mathbf{k}\right)_{\mathbf{k}_{n}}$ are the Fermi and "gap" velocities, and $\tilde{\mathbf{k}}$ is the momentum measured from the node at $\mathbf{k}_{n}$. Gapless nodal excitations determine the low-energy behavior of the clean DOS, $\nu_{c l}(\epsilon \rightarrow 0)=\alpha \epsilon$, where $\alpha=N /\left(2 \pi v_{F} v_{g}\right)$ and $N$ is the number of nodes.

Self-consistent T-matrix approximation. In sharp contrast with a normal metal, the DOS in $d$-wave superconductors is strongly affected by disorder. The commonly used self-consistent T-matrix approximation (SCTMA) yields finite DOS at the Fermi level, $\nu_{0}=\frac{2}{\pi} \alpha \gamma l$ (see, e.g., [11]). Here $\gamma$ is the impurity-induced relaxation rate of nodal excitations, $l=\ln (\Lambda / \gamma)$, and $\Lambda \sim\left(v_{F} v_{g}\right)^{1 / 2} a^{-1}$ is the high-energy cut-off in the nodal expansion.

Let us outline first the SCTMA for a general asymmetric band. Assuming that both the time-reversal and spinrotational symmetries are preserved, consider a random distribution of point-like spinless scatterers of arbitrary strength $U$ and concentration $n_{i}$. The self-consistent T- 
matrix $\hat{T}(\tilde{\epsilon})=\sum_{i} T_{i}(\tilde{\epsilon}) \hat{\tau}_{i}$, depicted in Fig. 11(a), has two Nambu components given by $T_{3}(\tilde{\epsilon}) \pm T_{0}(\tilde{\epsilon})=\left[c \mp g_{0}(\tilde{\epsilon})\right]^{-1}$, with $c=U^{-1}-g_{3}(\epsilon=0)$ and $\tilde{\epsilon}=\epsilon-\Sigma_{0}$. Here $\hat{\tau}_{1,2,3}$ are the Pauli matrices and $\hat{\tau}_{0}$ is the unity matrix, $\Sigma_{0}=n_{i} T_{0}$, and the chemical potential shift is $\delta \mu=\Sigma_{3}=n_{i} T_{3}$. The quantities $g_{0,3}$ are the Nambu components of the integrated Green's function $\hat{g}(\tilde{\epsilon})=\sum_{\mathbf{k}} \hat{G}_{\mathbf{k}}(\tilde{\epsilon})$, where $\hat{G}_{\mathbf{k}}(\tilde{\epsilon})=\left(\tilde{\epsilon} \hat{\tau}_{0}+\Delta_{\mathbf{k}} \hat{\tau}_{1}+\xi_{\mathbf{k}} \hat{\tau}_{3}\right) /\left(\tilde{\epsilon}^{2}-\varepsilon_{\mathbf{k}}^{2}\right) . \quad$ The above, closed system of equations apply to both the retarded (R) and advanced (A) channels, and it is understood that $\epsilon$ lies in the upper or lower half-plane as appropriate. We remark that the quantity $g_{3}(\epsilon=0)$ is real and vanishes for a perfectly symmetric band; it therefore cannot be estimated in a nodal expansion. At the level of SCTMA, $g_{3}$ just renormalizes the effective scattering potential with $U \rightarrow c^{-1}$. The Born and unitarity limits correspond to $c \gg\left|g_{0}\right|$ and $c \rightarrow 0$ respectively. The QP self-energies (QPSEs) are non-singular at small frequencies $\epsilon<\gamma$ where they obtain the form $\Sigma_{0}^{R(A)}(\epsilon)=\lambda \epsilon \mp i \gamma$. Here the relaxation rate $\gamma$ is the solution (cf. [11, 12]) of $\gamma=i n_{i} T_{0}(\epsilon=0)$, and the mass renormalization satisfies $\lambda=\left.n_{i} \partial_{\epsilon} T_{0}(\tilde{\epsilon})\right|_{\epsilon=0}$.

Analysis of diffusion modes. Since the SCTMA is exact only for a single-impurity problem, $\nu_{0}$ is subject to further corrections caused by multiple impurity scattering which result in new low-energy regimes [i] 6 ]. We explore these regimes by calculating the WL correction to the SCTMA result in the diffusion approximation. We observe that the first-order correction predicts many aspects of the low-energy behavior and qualitatively agrees with numerical calculations. In particular, the sign of the correction can be associated with the further tendency for $\nu(\epsilon \rightarrow 0)$ : to diverge (positive sign), to vanish (negative sign), or to saturate (correction is absent).

Consider the diffuson $\hat{\mathcal{D}}_{\mathbf{q}}\left(\epsilon, \epsilon^{\prime}\right)$ and Cooperon $\hat{\mathcal{C}}_{\mathbf{q}}\left(\epsilon, \epsilon^{\prime}\right)$ ladder diagrams depicted in Fig. 11(b). The 2-particle irreducible vertex $\hat{\mathcal{I}}_{\tilde{\epsilon}, \tilde{\epsilon}^{\prime}}=n_{i} \hat{T}(\epsilon) \otimes \hat{T}\left(\epsilon^{\prime}\right)$. The solution of the matrix equations for $\hat{\mathcal{D}}$ and $\hat{\mathcal{C}}$ is simplified if we make the decomposition $\mathcal{X}_{a b c d}=\frac{1}{2} \sum_{i j} \mathcal{X}^{i j} \tau_{i a d} \tau_{j c b}$ for $\mathcal{X}=\mathcal{I}, \mathcal{D}, \mathcal{C}$. In this basis, the ladder diagrams reduce to a $4 \times 4$ matrix equation. Furthermore, it is easy to show that $\mathcal{C}_{\mathbf{q}}^{i j}\left(\epsilon, \epsilon^{\prime}\right)=\mathcal{D}_{\mathbf{q}}^{i j}\left(\epsilon, \epsilon^{\prime}\right)$, and that only the diagonal components of the diffusive modes may be singular,

$$
\mathcal{D}_{\mathbf{q}}^{i i}\left(\epsilon, \epsilon^{\prime}\right)=\frac{\mathcal{I}_{\epsilon, \epsilon^{\prime}}^{i i}}{1-\frac{1}{2} \mathcal{I}_{\epsilon, \epsilon^{\prime}}^{i i} \sum_{\mathbf{k}} \operatorname{Tr}\left[\hat{G}_{\mathbf{k}+\mathbf{q}}(\tilde{\epsilon}) \hat{\tau}_{i} \hat{G}_{\mathbf{k}}\left(\tilde{\epsilon}^{\prime}\right) \hat{\tau}_{i}\right]},
$$

where $\mathcal{I}_{\epsilon, \epsilon^{\prime}}^{i j}=\frac{1}{2} n_{i} \operatorname{Tr}\left[\hat{T}(\tilde{\epsilon}) \hat{\tau}_{i} \hat{T}\left(\tilde{\epsilon}^{\prime}\right) \hat{\tau}_{j}\right]$. Besides the singular contributions, diffusive ladders contain the regular ones. For superconductors, the need to sum up the nonsingular ladders has been pointed out in Ref. [13].

In the RA channel, $\mathcal{D}^{00}$ and $\mathcal{C}^{00}$ have diffusive poles at small $\mathbf{q}$ and $\epsilon-\epsilon^{\prime}$,

$$
\mathcal{D}_{\mathbf{q}}^{00}\left(\epsilon, \epsilon^{\prime}\right)^{R A}=\mathcal{C}_{\mathbf{q}}^{00}\left(\epsilon, \epsilon^{\prime}\right)^{R A}=\frac{4 \gamma^{2}}{\pi \nu_{0}} \frac{1}{D_{0} q^{2}-i\left(\epsilon-\epsilon^{\prime}\right)} .
$$

(a)

(b)

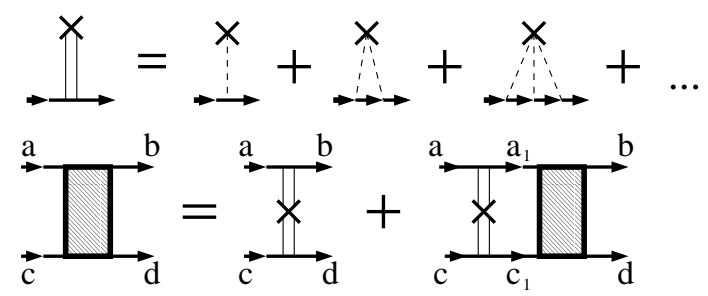

(c)

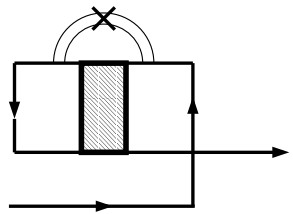

(d)

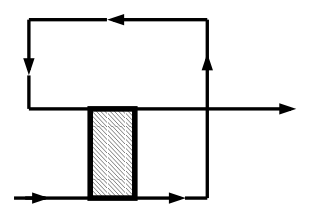

FIG. 1. (a) Quasiparticle self-energy in the self-consistent T-matrix approximation; (b) Diagramatic equation for the Cooperon $\mathcal{C}$ (the direction of the arrows in the bottom line should be reversed for the diffuson $\mathcal{D}$ ); (c) diffuson and (d) Cooperon contributions to DOS. Solid lines are quasiparticles, and dashed lines indicate impurity potentials $U$.

For diffusons and Cooperons, $\mathbf{q}$ is the difference and sum of momenta of QPs in the top and bottom lines in Fig. 1(b) respectively, and the diffusion coefficient $D_{0}$ is found to be $D_{0}=\bar{v}^{2} /(2 \gamma l)$ with $\bar{v}^{2}=\frac{1}{2}\left(v_{F}^{2}+v_{g}^{2}\right)$. The logarithmic factor $l$ in the denominator of $D_{0}$ originates from renormalization of the real part of the QPSE. Upon this renormalization, $D_{0}$ and $\nu_{0}$ obey the Einstein relation $D_{0} \nu_{0}=\sigma_{s} / s^{2}=\frac{1}{\pi} \alpha \bar{v}^{2}$, where $\sigma_{s}$ is the universal value of the Drude spin conductance [2, 11], and $s=\frac{1}{2}$ is the spin of electron.

Due to the intrinsic particle-hole symmetry of a superconductor, $\hat{\tau}_{2} \hat{G}_{\mathbf{k}}(-\tilde{\epsilon}) \hat{\tau}_{2}=-\hat{G}_{\mathbf{k}}(\tilde{\epsilon})$, diffusive modes also exist in the RR and AA channels 13]. These anomalous modes appear in $\mathcal{D}^{22}$ and $\mathcal{C}^{22}$ when $\epsilon, \epsilon^{\prime}<\gamma$,

$$
\mathcal{D}_{\mathbf{q}}^{22}\left(\epsilon, \epsilon^{\prime}\right)^{R R}=\mathcal{C}_{\mathbf{q}}^{22}\left(\epsilon, \epsilon^{\prime}\right)^{R R}=-\mathcal{D}_{\mathbf{q}}^{00}\left(\epsilon,-\epsilon^{\prime}\right)^{R A} .
$$

They are always gapless due to the fact that the spin and the energy are conserved quantities in the problem.

$W L$ correction in generic case. In the absence of special symmetries, Eq. (2) gives the only diffusive modes which contribute to the DOS. The first-order WL correction can be written in the form

$$
\delta \nu(\epsilon)=-\frac{1}{\pi} \operatorname{Im} \sum_{\mathbf{k}} \operatorname{Tr}\left[\hat{G}_{\mathbf{k}}^{R}(\tilde{\epsilon}) \hat{\Sigma}_{\mathbf{k}}^{R}(\tilde{\epsilon}) \hat{G}_{\mathbf{k}}^{R}(\tilde{\epsilon})\right]
$$

The skeleton diagrams for the self-energy $\hat{\Sigma}_{\mathbf{k}}^{R}(\tilde{\epsilon})$ are depicted in Figs. 11(c) and (d), with solid grey blocks denoting diffuson or Cooperon propagators. Diagram 1 (c) - the diffuson with one leg decorated by an irreducible vertex, as well as a similar diagram with the other leg decorated - vanishes, so the leading order contribution is the Cooperon diagram, $\mathbb{1}(\mathrm{d})$, which yields the negative logarithmic correction (as found in [2]14]) 


$$
\delta \nu(\epsilon)=-\frac{1}{4 \pi^{2} D_{0} l} \ln \frac{\gamma}{2 \epsilon} .
$$

This holds for generic bands and arbitrary strength of disorder. Since $D_{0} l=\bar{v}^{2} /(2 \gamma)$, the high-energy cut-off $\Lambda$ enters Eq. (四) via the scattering rate $\gamma$ only. Furthermore, the insertion of nonsingular ladders into diagrams Fig. 1(c)-(d) in all possible ways leads to ballistic renormalization of the parameter $D_{0}$ in Eq. (四). After the renormalization, $D_{0} \rightarrow D$, this expression is the complete singular contribution to the DOS in first order of perturbation theory in the inverse spin conductance $\sigma_{s}^{-1}$.

The supression of the DOS relative to $\nu_{0}$ at low energies indicated by Eq. (4) is seen in nearly all published numerical work [8,10,15, as well in most cases presented in Fig. 2. A glance at Fig. 2(b)-(e) shows that, although $\nu(\epsilon \rightarrow 0) \rightarrow 0$ in these cases, there is typically more structure than is contained in Eq. (4). In order to understand this better, we consider the effects of certain nesting symmetries.

Global nesting symmetry $\hat{\tau}_{2}$. The above Goldstone modes Eq. (2) occur for arbitrary renormalized potential $c^{-1}$. As it is seen from Eq. (11), additional pseudoGoldstone modes appear in the unitarity limit for systems which satisfy a GNS,

$$
\hat{\tau}_{2} \hat{G}_{\mathbf{k}} \hat{\tau_{2}}=\hat{G}_{\mathbf{k}+\mathbf{Q}}
$$

When Eq. (5) is exact and $c=0$ (e.g., in the $\mu=0$, $U=\infty \mathrm{N} 1$ model studied in $[6]$ ) the propagators $\mathcal{D}_{\mathbf{q}}$ and $\mathcal{C}_{\mathbf{q}}$ have additional poles at momenta close to $\mathbf{Q}=(\pi, \pi)$. These modes are related to the usual ones by

$$
\begin{aligned}
& \mathcal{D}_{\mathbf{Q}+\mathbf{q}}^{22}\left(\epsilon, \epsilon^{\prime}\right)^{R A}=\mathcal{C}_{\mathbf{Q}+\mathbf{q}}^{22}\left(\epsilon, \epsilon^{\prime}\right)^{R A}=\mathcal{D}_{\mathbf{q}}^{00}\left(\epsilon, \epsilon^{\prime}\right)^{R A} \\
& \mathcal{D}_{\mathbf{Q}+\mathbf{q}}^{00}\left(\epsilon, \epsilon^{\prime}\right)^{R R}=\mathcal{C}_{\mathbf{Q}+\mathbf{q}}^{00}\left(\epsilon, \epsilon^{\prime}\right)^{R R}=\mathcal{D}_{\mathbf{q}}^{22}\left(\epsilon, \epsilon^{\prime}\right)^{R R} .
\end{aligned}
$$

However, these modes are gapped by any distortion of the band which destroys the nesting symmetry or by the choice of nonunitarity potential $(c \neq 0)$, as we now show.

Firstly, we note that the N1 model at $\mu, c=0$ satisfies the GNS conditions exactly, so the $\pi$-modes are gapless in this case. Deviation from the half-filling $(\mu \neq 0)$ creates a gap which can be estimated in the nodal approximation as $\delta \approx 2 \mu^{2} /(\gamma l)$, whereas deviation from unitarity $(c \neq 0)$ yields $\delta \approx \gamma\left(4 c / \pi \nu_{0}\right)^{2}$. Secondly, the N2 model has nodes at $( \pm \pi / 2, \pm \pi / 2)$ as $\mu=0$, and the symmetry relation (5) is approximately satisfied near the nodal points. However, this is not the case in the entire Brillouin zone where the $t^{\prime}$-term evidently violates Eq. (5). As a result, the momentum regions located far away from the nodal points (these are momenta responsible for the global band asymmetry) contribute to a gap $\delta \propto t^{\prime 2}$ in $\mathrm{N} 2$ model even at $c=0$. We stress that this contribution cannot be estimated in the nodal approximation. Therefore, we conclude that the GNS relation (5) must hold througout the Brillouin zone, and not just near the gap

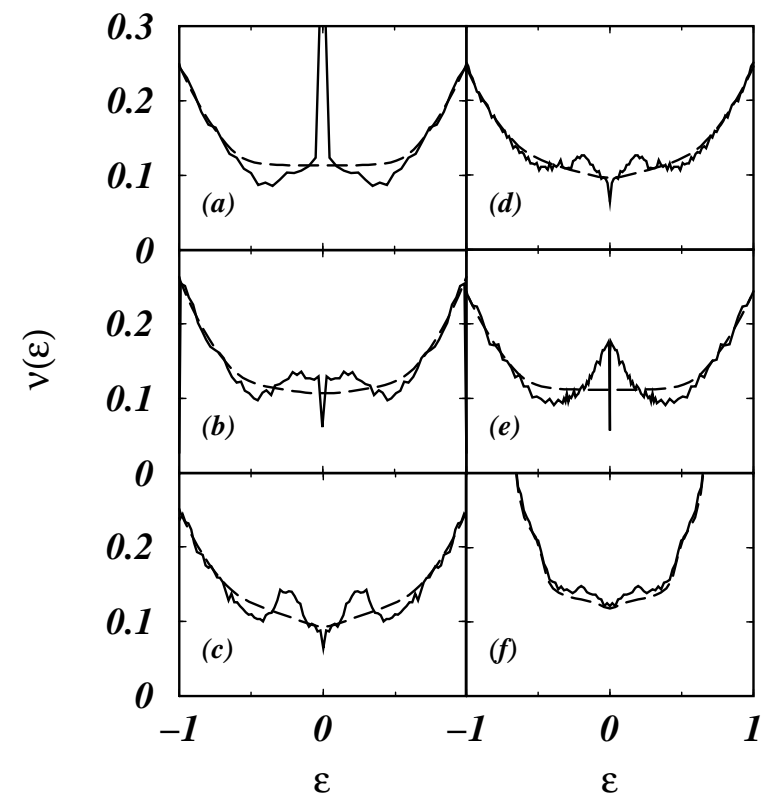

FIG. 2. Numerical results for $n_{i}=0.06$ and $\Delta_{0}=0.8$. Solid curves are solutions of BdG equations on a $30 \times 30$ lattice [except for (f), which is on a $45 \times 45$ lattice], dashed curves are SCTMA results. Curves are N1 with $\{\mu, U, c\}=($ a) $\left\{0,10^{4}, 10^{-4}\right\}$, (b) $\left\{0.6,10^{4}, 0.07\right\}$, (c) $\{0,10,0.10\}$; N2 with (d) $\left\{0,10^{4}, 0.099\right\}$, (e) $\left\{0,-11.675,-8 \times 10^{-5}\right\}$, and N3 with (f) $\{0,10,0.15\}$. Energies are measured in units of $t$ for N1 and N2 models, and $t^{\prime \prime}$ for the N3 model. The value of $t^{\prime}$ for $\mathrm{N} 2$ model is chosen to be 0.25 .

nodes; both deviations from Eq. (5) and deviations from unitarity gap the $\pi$-modes.

$W L$ correction in $\hat{\tau}_{2}$ case. The $\pi$-mode Cooperon makes a logarithmic contribution to $\delta \nu(\epsilon)$ of equal magnitude to Eq. (4) but - because it appears with different Nambu components - of opposite sign. The diffuson in Fig. If(c) makes a positive logarithmic contribution so the overall tendency is for $\delta \nu(\epsilon)$ to be positive. Thus, the additional symmetry (5) changes the asymptotic behavior from vanishing to divergent. If the $\pi$-modes are gapped but $\delta<\gamma$, then we identify two regimes: $\epsilon>\delta / 2$ where gapless ordinary modes and the gapped $\pi$-modes contribute on equal footing such that the total correction increases, and $\epsilon<\delta / 2$ where the $\pi$-mode contribution saturates whereas the ordinary one continue to suppress the DOS. Generally, the sum of these two terms can be written as follows

$$
\delta \nu(\epsilon)=\frac{1}{4 \pi^{2} D_{0} l}\left(-\ln \frac{\gamma}{2 \epsilon}+5 \ln \frac{\gamma}{\sqrt{4 \epsilon^{2}+\delta^{2}}}\right) .
$$

Numerical calculations [8, 10, 15], as well as nonperturbative analytical work on the half-filled N1 model [6] support this result. Fig. 2(a) shows a clear peak at $\epsilon=0$ for the N1 model with $\mu, c=0$. In Figs. 2(b)-(e) the $\pi$-modes are gapped in three different ways. In all cases, the qualitative behavior is the same: there is an initial 
upturn starting at $\epsilon \simeq \gamma$, which then crosses over to a downturn. As we move farther from the symmetric unitarity limit, either by increasing $\mu$, decreasing $U$ or increasing second-neighbour hopping, the peaks are shifted to higher energy, then become less pronounced, and after that disappear. In (b)-(e), the shape of the curves is qualitatively what one might expect based on Eq. (6). While this expression regularly overestimates the position of the maxima predicted as $\epsilon \sim \delta / 4$ (cf. Fig. 2), it is clear that the qualitative explanation based on the first-order perturbation correction could not pretend to describe all the details of the numerical experiment. We mention finally that the numerics [see Fig. 2(e)] and our WL calculation are in agreement that it is not possible to produce a divergence by means of fine-tuning parameters in the non-nested case, as suggested in Ref. [7].

Models with other nesting symmetries. There is a second possible nesting condition,

$$
\hat{\tau}_{3} \hat{G}_{\mathbf{k}} \hat{\tau_{3}}=\hat{G}_{\mathbf{k}+\mathbf{Q}}
$$

which leads to a non-generic DOS. A model (N3) with this symmetry which yields an exact solution was recently discussed in [П]. In this instance it was found, at least for Lorentzian disorder, that $\nu(\epsilon)$ remains finite as $\epsilon \rightarrow 0$. A similar result is seen in the WL calculations. While the 0 -modes are unaffected by Eq. (更), the $\pi$-modes change drastically. Divergences now appear in $\mathcal{D}^{11}$ and $\mathcal{D}^{33}$ with

$$
\begin{aligned}
& \mathcal{D}_{\mathbf{Q}+\mathbf{q}}^{33}\left(\epsilon, \epsilon^{\prime}\right)^{R A}=\mathcal{C}_{\mathbf{Q}+\mathbf{q}}^{33}\left(\epsilon, \epsilon^{\prime}\right)^{R A}=\mathcal{D}_{\mathbf{q}}^{00}\left(\epsilon, \epsilon^{\prime}\right)^{R A}, \\
& \mathcal{D}_{\mathbf{Q}+\mathbf{q}}^{11}\left(\epsilon, \epsilon^{\prime}\right)^{R R}=\mathcal{C}_{\mathbf{Q}+\mathbf{q}}^{11}\left(\epsilon, \epsilon^{\prime}\right)^{R R}=\mathcal{D}_{\mathbf{q}}^{22}\left(\epsilon, \epsilon^{\prime}\right)^{R R} .
\end{aligned}
$$

In this case, the diffusive modes contribute for arbitrary c. Calculating the WL correction to the DOS we observe, as before, the exact cancellation between 0 - and $\pi$-Cooperons. However, the contribution of $\pi$-diffusons [Fig. [1 (c)] now also sums to zero, and the total first-order WL correction to DOS is $\delta \nu(\epsilon)=0$.

The N3 model explicitly satisfies the symmetry (7) for arbitrary $\mu$, and a typical case is shown in Fig. 2(f). As expected, the DOS is finite at $\epsilon=0$, and agrees closely with the SCTMA result. This resembles the situation in a noninteracting normal metal [3], where the disorder by itself does not affect the DOS [16]. On the other hand, this model exhibits a Meissner effect. It is clear, however, that Eq. (7) describes a specific situation which is probably unrelated to the real cuprates [8]. We note that the approach of Ref. [7] fails to predict the characteristic behaviors of the $\hat{\tau}_{2}$ and $\hat{\tau}_{3}$ models identified here.

In order to complete our analysis of various extra symmetry possibilities, let us mention the purely artificial case: $\hat{\tau}_{1} \hat{G}_{\mathbf{k}} \hat{\tau}_{1}=\hat{G}_{\mathbf{k}+\mathbf{Q}}$. Then the gapless $\pi$-modes appear in the unitarity regime only, for which case we predict the constant $\nu(\varepsilon \rightarrow 0)$, since 0 - and $\pi$-Cooperons cancel each other whereas the diffuson contributions sum to zero.

In conclusion, we investigated the low-energy density of states in a disordered $2 \mathrm{D} d$-wave superconductor and argued that the generic feature of DOS is its lowenergy suppression. However, due to proximity to perfect nesting, pseudo-Goldstone diffusive mode with momenta close to $(\pi, \pi)$ gives rise to an energy dependence of DOS which can be strongly non-monotonic. The physics of this novel mode appears to account for the differences between many recent nonperturbative treatments and explains numerical results. The effects we predict should be visible in low-temperature specific heat experiments on disordered cuprates. In layered compounds with weak interlayer coupling $t_{\perp}$, WL corrections to the DOS appear for $t_{\perp}<\gamma$, but saturate for $\epsilon<t_{\perp}^{2} / \gamma$. Thus the criterion for a pronounced energy window in which WL corrections occur is $t_{\perp}^{2} / \gamma<\epsilon<\gamma$. This regime should be accessible in underdoped highly disordered materials.

The authors thank D. Diakonov, I. Gruzberg, I. Lerner, C. Mudry, C. Pépin, S. Sachdev, and A. Shytov for useful discussions. AGY, IVG, and DVK are grateful to NORDITA for hospitality. This work was supported by NSF Grants DMR-9974396 and INT-9815833 (WAA and PJH), DMR-9703388 (AGY), DMR-0071362 (DVK), by RFBR (IVG), and in part by INTAS.

$\dagger$ Also at Petersburg Nuclear Physics Institute, Gatchina, St. Petersburg 188300, Russia.

$\ddagger$ Also at A.F. Ioffe Physical-Technical Institute, St. Petersburg 194021, Russia.

[1] A.A. Nersesyan, A.M. Tsvelik, and F. Wenger, Phys. Rev. Lett. 72, 2628 (1994); Nucl. Phys. B 438, 561 (1995).

[2] T. Senthil, M.P.A. Fisher, L. Balents, and C. Nayak, Phys. Rev. Lett. 81, 4704 (1998); T. Senthil and M.P.A. Fisher, Phys. Rev. B 60, 6893 (1999).

[3] M. Bocquet, D. Serban, M.R. Zirnbauer, Nucl. Phys. B 578, 628 (2000); A. Altland, B.D. Simons, and M.R. Zirnbauer, cond-mat/0006362.

[4] K. Ziegler, M.H. Hettler, and P.J. Hirschfeld, Phys. Rev. Lett. 77, 3013 (1996); Phys. Rev. B 57, 10825 (1998).

[5] B. Huckenstein and A. Altland, cond-mat/0007413.

[6] C. Pépin and P.A. Lee, Phys. Rev. Lett. 81, 2779 (1998); Phys. Rev. B 63, 054502 (2001).

[7] C. Chamon and C. Mudry, Phys. Rev. B 63, 100503 (2001).

[8] W.A. Atkinson, P.J. Hirschfeld, A.H. MacDonald, and K. Ziegler, Phys. Rev. Lett. 85, 3926 (2000).

[9] Recently, the influence of $\pi$-modes on the $1 / 2$-filled normal metal was discussed in: E.P. Nakhmedov, M. Kumru, R. Oppermann, Phys. Rev. Lett. 84, 3930 (2000); E.P. Nakhmedov, H. Feldmann, R. Oppermann, and M. Kumru, Phys. Rev. B 62, 13490 (2000); see however: I. Gruzberg, A. Shytov and P.J. Hirschfeld, condmat/0102197. 
[10] W.A. Atkinson, P.J. Hirschfeld, and A.H. MacDonald, Phys. Rev. Lett. 85, 3922 (2000).

[11] A.C. Durst and P.A. Lee, Phys. Rev. B 62, 1270 (2000).

[12] P.A. Lee, Phys. Rev. Lett. 71, 1887 (1993).

[13] A. Altland and M.R. Zirnbauer, Phys. Rev. B 55, 1142 (1997).

[14] D.V. Khveshchenko, A.G. Yashenkin, and I.V. Gornyi,
Phys. Rev. Lett. 86, 4668 (2001).

[15] J.-X. Zhu, D.N. Sheng, and C.S. Ting, Phys. Rev. Lett. 85, 4944 (2000); A. Ghosal, M. Randeria, and N. Trivedi, Phys. Rev. B 63, R020505 (2001).

[16] B.L. Altshuler and A.G. Aronov, in Electron-Electron Interactions in Disordered Systems, edited by A.L. Efros and M. Pollak (Elsevier, New York, 1985). 